\title{
Evaluación de la calidad durante el almacenamiento de nueces Pecán [Carya illinoinensis (Wangenh.) C. Koch] acondicionadas en diferentes envases
}

\author{
Por Tatiana Oro ${ }^{1}$, Paulo José Ogliari ${ }^{1}$, Renata Dias de Mello Castanho Amboni ${ }^{1}$, \\ Daniel Barrera-Arellano ${ }^{2}$ y Jane Mara Block ${ }^{1}$ * \\ ${ }^{1}$ Centro de Ciências Agrárias, Departamento de Ciência de Alimentos, UFSC - \\ Florianópolis - SC, Brasil. ${ }^{2}$ Faculdade de Engenharia de Alimentos, \\ Departamento de Tecnologia de Alimentos - UNICAMP, Campinas - SP, Brasil. \\ E-mail de contacto: jmblock@cca.ufsc.br
}

\section{RESUMEN}

Evaluación de la calidad durante el almacenamiento de nueces Pecán [Carya illinoinensis (Wangenh.) C. Koch] acondicionadas en diferentes envases.

En el presente trabajo, fueron evaluadas las alteraciones en la calidad de nueces Pecán almacenadas en película plástica de nilón-polietileno al vacío y en recipientes plásticos de polipropileno, en temperatura ambiente, durante 150 días. La composición nutricional, en ácidos grasos $(62,5 \%$ de ácido oleico) y el contenido de tocoferoles $(30 \mathrm{mg} / \mathrm{kg}$ de $\gamma$-tocoferol) de las nueces Pecán, indican características nutricionales interesantes. Durante el almacenamiento de las nueces, el contenido de humedad no sufrió cambios significativos, mas ocurrió un oscurecimiento gradual y significativo de la superficie de las nueces. Las alteraciones en los índices de acidez $(0,17-0,37$ y $0,19-0,57$ inicial y final para los envases de película plástica y polipropileno, respectivamente), peróxido $(1,0-2,7$ y $1,1-4,7)$ y extinción específica a $232 \mathrm{~nm}(0,98-1,99$ y $0,96-2,16)$ y $270 \mathrm{~nm}(0,11-0,33$ y $0,04-0,15)$ en el aceite extraído fueron significativas. La calidad microbiológica fue excelente y no fue detectada la presencia de Salmonella sp. A través del análisis sensorial se determinó la vida de anaquel del producto como 120 días, sin diferencias significativas entre los dos tipos de envases utilizados.

PALABRAS-CLAVE: Calidad - Carya illinoinensis - Nuez Pecán - Vida de anaquel.

\section{SUMMARY}

Quality evaluation of Pecan nuts [Carya illinoinensis (Wangenh.) C. Koch] during storage in different packaging.

In the present work, the quality changes of pecan nuts stored in nylon-polyethylene plastic film under vacuum and in polypropylene plastic recipients at ambient temperature for 150 days were evaluated. The nutritional composition, fatty acid composition and tocopherol content of the pecan nuts reveal interesting nutritional characteristics and an oil with high contents of mono-unsaturated fatty acids and $\gamma$-tocopherol. During storage, moisture content did not suffer significant changes, but a gradual and significant darkening of the surface of the nuts occurred. The changes in acid value, peroxide value and specific extinction of the extracted oil were significant. The microbiological quality was excellent and the presence of Salmonella sp. was not detected. Through sensory analysis, the shelf-life of the product was determined as 120 days, without significant differences between the two types of packaging used.

KEY-WORDS: Carya illinoinensis - Pecan nut - Quality - Shelf-life.

\section{INTRODUCCIÓN}

Las nueces Pecán [Carya illinoinensis (Wangenh.) K. Koch] son nativas de América del Norte y pueden ser encontradas desde Nebraska e lowa en los Estados Unidos hasta el sur de México (Venkatachalam, 2004).

La producción industrial de nueces fue iniciada hace más de cien años, siendo actualmente los Estados Unidos responsables por más de $80 \%$ de la producción mundial (Venkatachalam, 2004). Otros países productores incluyen México, Australia, Israel, Brasil y Argentina (Ortiz, 2000).

Aunque Brasil es un importante productor de nueces, como nuez de Pará ("Brazil nut"), nuez de la India ("Cashew nut") y nuez de Macadamia ("Australian nut"), la producción de nuez Pecan está concentrada en la región Sur (Estados de Rio Grande do Sul, Santa Catarina, Paraná y São Paulo). Según los datos del Ministerio de Agricultura, se producen anualmente en Brasil dos mil toneladas de nueces, con un rendimiento medio de 1.022 $\mathrm{kg} / \mathrm{ha}$ (Brasil, 2007).

Las nueces son muy apreciadas por su sabor y textura agradables, y son utilizadas (crudas, tostadas y/o saladas) en ensaladas, postres, helados, "snacks" y en productos de panadería y confitería. Las nueces son comercializadas con o sin cáscara, en mitades, en pedazos de diferentes tamaños o molidas (harina), y también pueden servir como materia-prima para la extracción de aceite.

Las nueces tienen un contenido elevado de lípidos, que alcanza hasta $75 \%$, dependiendo de la variedad, del local de producción, de la composición 
del suelo y de la época de cosecha. Como la mayoría de las nueces, las nueces Pecán contienen ácidos grasos de 16 y 18 carbonos. Aproximadamente $98 \%$ de los triacilglicéridos son compuestos por los ácidos palmítico, esteárico, oleico, linoleico y linolénico. Los ácidos grasos insaturados representan la mayor parte de la fracción lipídica, siendo el ácido oleico el ácido graso predominante (Venkatachalam, 2004). Algunos estudios sugieren que puede existir una relación entre el consumo frecuente de nueces y la reducción de la incidencia de enfermedades cardíacas coronarias (Crews et al., 2005; Kornsteiner et al. , 2006).

Alimentos ricos en lípidos son muy susceptibles a la oxidación y con frecuencia se tornan rancios durante el almacenamiento (Valenzuela y Nieto, 2001), resultado del desarrollo de sabores y olores desagradables, además de la destrucción de compuestos nutricionalmente importantes, como vitaminas liposolubles, ácidos grasos esenciales, carotenoides, aminoácidos, proteínas o enzimas. Este tipo de deterioración reduce la vida útil y compromete la integridad y seguridad de los alimentos, debido a la producción de compuestos fisiológicamente activos (Maskan y Karatas, 1999).

La oxidación lipídica se ve afectada por factores como la composición en ácidos grasos, contenido y actividad de pro y antioxidantes, radiación ultravioleta, temperatura, presencia de iones metálicos, presión de oxígeno, superficie de contacto con el oxígeno y actividad de agua (Kolakowska, 2003).

La calidad de las nueces y su estabilidad dependen de la composición inicial, además de las prácticas de manejo durante el cultivo y cosecha y de los métodos utilizados en el procesamiento, envase y almacenamiento. El envase representa un papel fundamental, una vez que representa una barrera para algunos de los factores involucrados en el proceso de oxidación y en la pérdida de calidad, como luz, oxígeno y vapor de agua.

Aunque existe un aumento en la producción y comercialización de nueces en Brasil, se conoce muy poco sobre las características nutricionales y estabilidad oxidativa de estos productos y sobre los cambios en su calidad físico-química, sensorial y microbiológica durante el almacenamiento.

El objetivo de este trabajo fue evaluar los cambios en la calidad de nueces a través de la determinación de las características físico-químicas del aceite extraído y características sensoriales y microbiológicas de las nueces, durante el almacenamiento por 150 días en envases plásticos de polipropileno y en películas plásticas de nilón-polietileno al vacío.

\section{MATERIAL Y MÉTODOS}

\subsection{Material}

Nueces Pecán [Carya illinoinensis (Wangenh.) $\mathrm{K}$. Koch] compuestas por una mezcla de las variedades: Barton (cerca de 50\%), Shoshone, Shaw- nee, Choctaw y Cape Fear, cosecha 2006, provenientes de varias propiedades rurales de la región de Cachoeira do Sul, RS, Brasil.

Las nueces en mitades fueron descascaradas, seleccionadas y envasadas por la empresa Divinut Indústria de Nozes Ltda., en película de nilón-polietileno al vacío y en recipientes plásticos de polipropileno, ambos con capacidad para $100 \mathrm{~g}$.

\subsection{Procedimiento Experimental}

\section{Determinación de la composición nutricional de las nueces}

La composición nutricional fue determinada de acuerdo con la metodología de la Association of Official Analytical Chemists (AOAC, 2005), a través de los siguientes análisis: humedad $(925,09)$; proteínas $(920,87)$; lípidos totales $(920,85)$; fibras totales, solubles e insolubles $(991,43)$ y minerales $(923,03)$; los carbohidratos fueron estimados por diferencia. La composición en ácidos grasos y los tocoferoles fueron determinados por los métodos AOCS (2003) Ce 1-91 y Ce 8-89, respectivamente.

\section{Determinación de la calidad durante el almacenamiento}

Los envases conteniendo las nueces fueron mantenidos en local con temperatura (media de $\left.23,1 \pm 2,1^{\circ} \mathrm{C}\right)$ y humedad relativa $(55,6 \pm 4,5 \%)$ controladas por 150 días.

A cada 15 días, una porción de $50 \mathrm{~g}$ de nueces fue molida y prensada en una prensa hidráulica (TECNAL, modelo TE-098), obteniéndose el aceite crudo utilizado para la determinación de los siguientes parámetros relacionados con la calidad de las nueces, utilizando los métodos oficiales de la American Oil Chemists' Society (AOCS, 2003): humedad y compuestos volátiles (Ca 2c-25); índice de peróxido (Cd 8-53); índice de acidez (Cd 3d-63) y extinción específica a 232 y $270 \mathrm{~nm}$ (Ch 5-91). La estabilidad oxidativa fue estimada mediante el método OSI (Cd 12b-92) en el aceite extraído en el inicio del experimento.

Las determinaciones microbiológicas se realizaron a cada 30 días, siguiendo la metodología oficial da American Public Health Association (APHA, 2001) para recuento total de hongos y levaduras, coliformes a $45^{\circ} \mathrm{C}$ y Salmonella sp.

\section{Color}

El color de las nueces fue determinado a cada 30 días, de acuerdo con el sistema de evaluación de la CIE Lab (Comission International de l'Eclairage) utilizando un colorímetro marca Minolta Chromo Meter CR 400 (Minolta, Osaka, Japón), acoplado a un procesador DP-100, escala de color L, a y $\mathrm{b}$, donde $\mathbf{L}$ corresponde a la luminosidad $(0=$ negro a $100=$ blanco); a corresponde a la variación del color de verde a rojo (de -80 hasta cero $=$ verde, de 
cero $\mathbf{a}+100=$ rojo); y b corresponde a la variación del color de azul a amarillo (de -100 hasta cero $=$ azul, de cero a $+70=$ amarillo). Se calculó (seis repeticiones) el índice de oscurecimiento (IO), de acuerdo con la ecuación (Buera et al. , 1985):

$$
\mathrm{IO}=\frac{100(x \times 0,31)}{0,172}
$$

donde

$$
x=\frac{a+1,75 L}{5,645 L+a-3,012 b}
$$

\section{Análisis Sensorial}

Las nueces acondicionadas en los diferentes envases fueron evaluadas a cada 30 días utilizándose una escala hedónica estructurada de 9 puntos (1 = me disgustó muchísimo; 9 = me gustó muchísimo) (Meilgaard et al. , 1999). La nota 6,0 de la escala fue definida como el límite de calidad 0 aceptabilidad (cut-off) (Muñoz et al., 1992). La aceptablidad sensorial fue evaluada en relación a apariencia, color, olor, sabor, textura e impresión global por 30 consumidores de nueces. Las muestras fueron evaluadas en cabines individuales con luz blanca y enseguida con luz roja, para ocultar el color. Las muestras de cerca de $5 \mathrm{~g}$ fueron servidas a los evaluadores de forma monádica y balanceada con números de 3 dígitos aleatorios.

\section{Análisis Estadístico}

El análisis estadístico fue realizado utilizando el programa Statistica, Versión 6.0, con un nivel de confianza de $p<0,05$. Se efectuó análisis de correlación lineal simples para todas las variables analizadas para cada tratamiento y entre los tratamientos a través del tiempo. Para verificar la dependencia entre las variables fue utilizada la correlación de Pearson. El análisis de contrastes fue realizado para verificar las diferencias estadísticas entre los tratamientos.

\section{RESULTADOS Y DISCUSIÓN}

\subsection{Composición nutricional}

En la Tabla 1, se presentan los resultados de la composición de las nueces. De acuerdo con los resultados obtenidos, los lípidos representan la fracción principal (cerca de $70 \%$ ) de este tipo de nuez. Los valores obtenidos corresponden a valores reportados en la literatura, entre 60 y $75 \%$, dependiendo de la variedad, local y año de producción, composición del suelo y época de cosecha (Villarreal-Lozoya et al., 2007; Shahidi y Miraliakbari, 2005; Firestone, 1999). El contenido de proteínas (9,9\%) es similar al obtenido en nueces Pecán australianas, entre 9,0 y $10,4 \%$ (Singanusong et al., 2003). El porcentaje de minerales $(1,4 \%)$ es similar al re- portado por el Departamento de Agricultura de los Estados Unidos (USDA, 2007), de 1,5\%. El contenido de agua $(3,7 \%)$ está entre los valores ideales para la conservación de nueces, entre 3,5 y 4,0\% (Shahidi y Miraliakbari, 2005). Los resultados obtenidos para fibras totales $(7,8 \%)$ son superiores a los publicados por Wakeling et al. (2001) para nueces de las variedades Wichita e Western Schley, producidas en tres cosechas diferentes, que oscilaron entre 2,9 y $4,2 \%$. El valor calórico calculado, de 726,7 $\mathrm{kcal} / \mathrm{g}$, es ligeramente superior al reportado por Maskan y Karatas (1999), de 700 kcal/100 g.

En la Tabla 2, se muestra la composición en ácidos grasos del aceite extraído de las nueces Pecán, donde los ácidos grasos predominantes fueron los ácidos oleico (62,5\%) y linoleico $(27,5 \%)$. Estos resultados son similares a los reportados por Wakeling et al. (2001), para aceite de nueces Pecán, variedades Wichita y Western Schley, con valores medios de 55,33\% de ácido oleico y 32,88\% de linoleico. Villarreal-Lozoya et al. (2007) reportaron rangos de 53 a $75 \%$ para oleico y de 15 a $36 \%$ para linoleico en siete variedades producidas en los Estados Unidos.

El contenido de tocoferoles en el aceite fue de $30,0 \mathrm{mg} / 100 \mathrm{~g}$ de $\gamma$-tocoferol; ningún otro tocoferol fue detectado. Kornsteiner et al. (2006) indicaron valores de tocoferoles de $14,8 \mathrm{mg} / 100 \mathrm{~g} \beta$ - y $\gamma$-to-

Tabla 1

Composición nutricional $(\mathrm{g} / 100 \mathrm{~g})$ de nueces Pecán [Carya illinoinensis (Wangenh.) C. Koch]

\begin{tabular}{lc}
\hline \multicolumn{1}{c}{ Componente $(\mathbf{g} / \mathbf{1 0 0 g})^{1}$} & Nueces \\
\hline Proteínas $^{2}$ & $9,9 \pm 0,1$ \\
Humedad & $3,7 \pm 0,3$ \\
Lípidos Totales & $69,4 \pm 0,1$ \\
Minerales & $1,4 \pm 0,2$ \\
Fibra total & $7,8 \pm 0,45$ \\
Fibra soluble & $0,5 \pm 0,3$ \\
Fibra insoluble & $7,3 \pm 0,5$ \\
Carbohidratos $^{3}$ & 7,8 \\
Valor calórico $^{4}(\mathrm{kcal} / 100 \mathrm{~g})$ & 726,7 \\
\hline
\end{tabular}

${ }^{1}$ Media de tres repeticiones; ${ }^{2} \mathrm{~N} \times 6,25 ;{ }^{3}$ Calculado por diferencia: $100-$ (\% lípidos totales $+\%$ proteínas $+\%$ humedad $+\%$ minerales $+\%$ fibra total). ${ }^{4}$ Calculado por la suma de los porcentages de proteínas y carbohidratos multiplicados por el factor $4(\mathrm{kcal} / \mathrm{g})$ más el contenido de lípidos totales multiplicado por $9(\mathrm{kcal} / \mathrm{g})$.

Tabla 2

Composición e ácidos grasos del aceite de nuez Pecán [Carya illinoinensis (Wangenh.) C. Koch]

\begin{tabular}{lc}
\hline \multicolumn{1}{c}{ Ácido graso } & $\mathbf{( \% )}$ \\
\hline C 16:0 Palmítico & 5,65 \\
C 18:0 Esteárico & 2,84 \\
C 18:1 Oléico & 62,55 \\
C 18:2 Linoléico & 27,49 \\
C 18:3 Linolénico & 1,23 \\
C 20:1 Gadoléico & 0,24 \\
\hline
\end{tabular}


coferol y $0,2 \mathrm{mg} / 100 \mathrm{~g} \delta$-tocoferol en nueces de Austria y Grecia. Chun et al. (2002) reportaron valores de 22,0, 20,1, 23,9 y 29,3 mg/100 g de $\gamma$-tocoferol, para las variedades Seedling, Desirable, Stuart y Schley, respectivamente. EI USDA (2006) considera, en media, $2,53 \mathrm{mg} / 100 \mathrm{~g}$ de $\alpha$-tocoferol, $0,35 \mathrm{mg} / 100 \mathrm{~g}$ de $\beta$-tocoferol, $24,2 \mathrm{~g} / 100 \mathrm{~g}$ de $\gamma$-tocoferol y $1,07 \mathrm{~g} / 100 \mathrm{~g}$ de $\delta$-tocoferol. En este trabajo, además de solo haber detectado $\gamma$-tocoferol, la concentración fue superior a los valores encontrados en la literatura citada.

\subsection{Determinación de la calidad durante el almacenamiento}

En la Tabla 3, son mostrados los resultados obtenidos para el índice de acidez y la humedad y compuestos volátiles de las nueces almacenadas en película de nilón-polietileno al vacío y en recipientes plásticos durante 150 días, determinados en el aceite extraído de las nueces.

Los resultados para humedad variaron entre 0,04 y $0,07 \%$ y entre 0,08 y $0,06 \%$ para las nueces almacenadas en recipientes y película, respectivamente. Los valores máximos fueron alcanzados a los 90 días de almacenamiento. Fue observada una variación lineal no significativa $(p \geq 0,05)$ con respecto al tiempo, con una tendencia de aumento en los recipientes y de reducción en las películas al vacío. El análisis de contrastes indicó una diferencia significativa $(p<0,05)$ en el contenido de humedad entre los dos tipos de envases.

En relación al índice de acidez, fue observada una variación entre 0,17 y $0,37 \mathrm{mg} \mathrm{KOH} / \mathrm{g}$ para las nueces almacenadas en recipientes plásticos y entre 0,19 y $0,57 \mathrm{mg} \mathrm{KOH} / \mathrm{g}$ para aquellas almacenadas en películas al vacío. Esta variación en el tiempo fue estadísticamente significativa ( $p \leq 0,0001$, $R^{2}=0,8712$ y 0,7921 , para recipientes plásticos y película de nilón-polietileno al vacío, respectivamente), sin embargo el análisis de contrastes mostró que no hubo diferencias significativas debidas al tipo de envase $(p \geq 0,05)$.
Gamli y Hayoglu (2007) no encontraron diferencias significativas en el almacenamiento de pasta de pistachos en envases transparentes de polietileno, con y sin vacío, a 4 y $20^{\circ} \mathrm{C}$, por un período de 210 días.

En la Tabla 4, son mostrados los resultados para los parámetros índice de peróxido y extinción específica, relacionados a la calidad y medidos en el aceite extraído de las nueces almacenadas en película de nilón-polietileno al vacío y en recipientes plásticos de polipropileno por 150 días.

Los valores de índice de peróxido (IP) variaron entre 1,04 y 2,66 meq $\mathrm{O}_{2} / \mathrm{kg}$ y 1,14 y 4,67 meq $\mathrm{O}_{2} / \mathrm{kg}$ al inicio y después de 150 días de almacenamiento, para las nueces en película de nilón-polietileno al vacío y en recipientes plásticos de polipropileno, respectivamente.

El análisis de regresión indicó que el índice de peróxido cambió significativamente en el período de almacenamiento en los dos tipos de envases $\left(R^{2} \geq 0,5958\right.$ y $\left.p \leq 0,0089\right)$. Entre los tratamientos, se observó que la alteración del índice de peróxido en los envases con película al vacío fue superior al observado en los recipientes plásticos. Mas, el análisis estadístico de contrastes no indicó diferencias significativas entre los tratamientos $(p \geq 0,05)$.

García-Pascual et al. (2003) observaron que la atmósfera de almacenamiento (aire o nitrógeno) no tuvo efecto sobre la evolución del índice de peróxido en almendras envasadas en películas plásticas, a temperaturas de 8 y $36{ }^{\circ} \mathrm{C}$, durante 4 meses.

Con relación a la extinción específica a $232 \mathrm{~nm}$, los valores variaron entre 0,98 y 1,99 en los recipientes plásticos y entre 0,96 y 2,16 en las nueces almacenadas en película plástica; ambos tratamientos alcanzaron el valor máximo de 3,20 a los 120 días. Estos resultados indican que, después de 120 días de almacenamiento, ocurre una transformación de los compuestos primarios de oxidación en otros compuestos no detectables a $232 \mathrm{~nm}$. El coeficiente de extinción específica medido a $232 \mathrm{~nm}$

Tabla 3

Evolución de los parámetros de calidad, Índice de acidez y humedad y compuestos volátiles en el aceite extraído de nueces almacenadas en recipientes plásticos de polipropileno y en película de nilón-polietileno al vacío.

\begin{tabular}{ccccc}
\hline \multirow{2}{*}{ Tiempo (dias) } & \multicolumn{2}{c}{ Humedad $^{\mathbf{1}}$ (\%) } & \multicolumn{2}{c}{ IA $^{\mathbf{2}}$ (mg KOH/g de aceite) } \\
\cline { 2 - 4 } & Recipientes & Película & Recipientes & Película \\
\hline 0 & $0,04 \pm 0,01$ & $0,08 \pm 0,01$ & $0,17 \pm 0,01$ & $0,19 \pm 0,01$ \\
30 & $0,07 \pm 0,01$ & $0,07 \pm 0,01$ & $0,22 \pm 0,01$ & $0,31 \pm 0,01$ \\
45 & $0,07 \pm 0,02$ & $0,07 \pm 0,01$ & $0,21 \pm 0,01$ & $0,21 \pm 0,01$ \\
60 & $0,04 \pm 0,01$ & $0,05 \pm 0,01$ & $0,24 \pm 0,01$ & $0,23 \pm 0,01$ \\
75 & $0,07 \pm 0,02$ & $0,06 \pm 0,01$ & $0,25 \pm 0,01$ & $0,33 \pm 0,01$ \\
90 & $0,07 \pm 0,01$ & $0,07 \pm 0,01$ & $0,34 \pm 0,01$ & $0,32 \pm 0,01$ \\
105 & $0,07 \pm 0,01$ & $0,06 \pm 0,01$ & $0,35 \pm 0,01$ & $0,36 \pm 0,01$ \\
120 & $0,07 \pm 0,01$ & $0,06 \pm 0,01$ & $0,33 \pm 0,01$ & $0,44 \pm 0,02$ \\
135 & $0,06 \pm 0,01$ & $0,05 \pm 0,03$ & $0,37 \pm 0,00$ & $0,42 \pm 0,01$ \\
150 & $0,07 \pm 0,01$ & $0,06 \pm 0,01$ & $0,57 \pm 0,01$ \\
\hline
\end{tabular}

${ }^{1}$ Humedad y compuestos volátiles, media de tres repeticiones. ${ }^{2}$ Índice de acidez, media de dos repeticiones. 
Tabla 4

Evolución de los parámetros de calidad, índice de peróxido y extinción específica a $232 \mathrm{~nm}$ y $270 \mathrm{~nm}$ en el aceite extraído de nueces almacenadas en recipientes plásticos de polipropileno y en película de nilón-polietileno al vacío

\begin{tabular}{ccccccc}
\hline \multirow{2}{*}{ Tiempo (dias) } & \multicolumn{2}{c}{$\mathbf{I P}^{\mathbf{1}}$ (meq $\mathbf{O}_{\mathbf{2}} / \mathbf{k g}$ de aceite) } & \multicolumn{2}{c}{$\mathrm{EE}^{\mathbf{2}} \mathbf{2 3 2} \mathbf{~ n m}$} & \multicolumn{2}{c}{$\mathrm{EE}^{\mathbf{2}} \mathbf{2 7 0} \mathbf{n m}$} \\
\cline { 2 - 7 } & Recipientes & Película & Recipientes & Película & Recipientes & Película \\
\hline 0 & $1,0 \pm 0,1$ & $1,1 \pm 0,1$ & $0,98 \pm 0,01$ & $0,96 \pm 0,06$ & $0,11 \pm 0,00$ & $0,04 \pm 0,03$ \\
30 & $1,2 \pm 0,1$ & $1,9 \pm 0,1$ & $1,08 \pm 0,02$ & $1,20 \pm 0,08$ & $0,10 \pm 0,00$ & $0,13 \pm 0,00$ \\
45 & $2,1 \pm 0,1$ & $2,1 \pm 0,1$ & $1,28 \pm 0,04$ & $1,19 \pm 0,09$ & $0,27 \pm 0,01$ & $0,19 \pm 0,02$ \\
60 & $2,1 \pm 0,2$ & $2,3 \pm 0,1$ & $1,59 \pm 0,09$ & $1,37 \pm 0,00$ & $0,34 \pm 0,01$ & $0,17 \pm 0,02$ \\
75 & $2,8 \pm 0,1$ & $3,5 \pm 0,1$ & $1,53 \pm 0,22$ & $1,49 \pm 0,02$ & $0,25 \pm 0,03$ & $0,24 \pm 0,05$ \\
90 & $3,5 \pm 0,1$ & $3,5 \pm 0,1$ & $1,74 \pm 0,28$ & $1,91 \pm 0,07$ & $0,14 \pm 0,01$ & $0,31 \pm 0,03$ \\
105 & $3,8 \pm 0,1$ & $4,6 \pm 0,2$ & $1,41 \pm 0,06$ & $2,07 \pm 0,08$ & $0,14 \pm 0,02$ & $0,32 \pm 0,02$ \\
120 & $4,3 \pm 0,1$ & $5,9 \pm 0,1$ & $3,20 \pm 0,05$ & $3,20 \pm 0,03$ & $0,32 \pm 0,00$ & $0,27 \pm 0,07$ \\
135 & $3,1 \pm 0,2$ & $4,8 \pm 0,4$ & $2,49 \pm 0,05$ & $2,42 \pm 0,04$ & $0,18 \pm 0,00$ & $0,04 \pm 0,01$ \\
150 & $2,7 \pm 0,1$ & $4,7 \pm 0,1$ & $1,99 \pm 0,01$ & $2,16 \pm 0,06$ & $0,33 \pm 0,02$ & $0,15 \pm 0,05$ \\
\hline
\end{tabular}

${ }^{1}$ Índice de peróxido, media de dos repeticiones, ${ }^{2}$ Extinción específica, media de tres repeticiones.

mostró una variación lineal altamente significativa en los dos tipos de envases $\left(p \leq 0,0001\right.$ y $R^{2}=$ 0,5672 y $R^{2}=0,7110$, para las nueces almacenadas en recipientes y película al vacío, respectivamente). El analísis estadístico de contrastes indicó que las diferencias entre los envases no fueron significativas $(p \geq 0,05)$. Resultados similares fueron observados en la medición de la extinción específica a $270 \mathrm{~nm}$, siendo las diferencias entre los envases no significativas.

Los parámetros índice de peróxido y extinción específica a $270 \mathrm{~nm}$ mostraron una correlación significativa y positiva $\left(p \leq 0,0001\right.$ y $R^{2}=0,7405$ y $R^{2}=0,9524$, para las nueces almacenadas en recipientes plásticos y película de nilón-polietileno al vacío, respectivamente). Chun et al. (2005) reportaron una correlación positiva $(R=0,9809)$ entre el índice de peróxido y la extinción específica a 232 $\mathrm{nm}$ de maní almacenado por 40 semanas en envases con barrera metalizada de poliéster al vacío y con aire a temperatura de $21^{\circ} \mathrm{C}$. Alves et al. (2005) observaron una correlación altamente significativa ( $p<0,0001)$ entre el índice de peróxido y la extinción específica a 232 y $270 \mathrm{~nm}\left(R^{2}=0,82\right)$ en aceite de soja almacenado en envases de PET.

\subsection{Cambios en el color}

Las alteraciones en el color de las nueces almacenadas por 150 días, en los dos tipos de envase, están relacionados en la Tabla 5 .

El análisis de contrastes no reveló diferencias significativas entre los resultados obtenidos para los parámetros de color $L$, a y b entre los dos tipos de envases evaluados ( $p \geq 0,05)$. Algunos parámetros sufrieron alteraciones a lo largo del tiempo. El parámetro $\mathbf{L}$, que corresponde a la luminosidad, mostró valores significativamente decrecientes en los dos envases ( $p \leq 0,0068$ y $\left.R^{2} \geq 0,868\right)$, con el consecuente oscurecimiento gradual y continuo de la superficie de las nueces durante todo el período.

El parámetro a, que representa el color rojo, no presentó diferencias significativas $(p \geq 0,05)$. El parámetro $\mathbf{b}$, que indica el color amarillo, disminuyó significativamente ( $p \leq 0,016$ y $\left.R^{2} \geq 0,8009\right)$, al mismo tiempo que el índice de oscurecimiento aumentó significativamente en los dos tipos de envases $\left(p \leq 0,0348\right.$ y $\left.R^{2} \geq 0,7116\right)$. Forbus et al. (1980) reportaron la reducción lineal de los parámetros $L$ y b durante el almacenamiento de nueces. Erickson et al. (1994) relataron alteraciones

Tabla 5

Evaluación instrumental de las alteraciones en el color de nueces almacenadas en recipientes plásticos de polipropileno y en película de nilón-polietileno al vacío

\begin{tabular}{|c|c|c|c|c|c|c|c|c|}
\hline \multirow{2}{*}{$\begin{array}{l}\text { Tiempo } \\
\text { (días) }\end{array}$} & \multicolumn{2}{|c|}{ L } & \multicolumn{2}{|c|}{ a } & \multicolumn{2}{|c|}{ b } & \multicolumn{2}{|c|}{$10^{1}$} \\
\hline & $\mathbf{R}$ & $\mathbf{P}$ & $\mathbf{R}$ & $\mathbf{P}$ & $\mathbf{R}$ & $\mathbf{P}$ & $\mathbf{R}$ & $\mathbf{P}$ \\
\hline 0 & $38,0 \pm 2,6$ & $36,1 \pm 4,2$ & $11,67 \pm 1,5$ & $9,2 \pm 0,8$ & $17,5 \pm 1,1$ & $15,9 \pm 1,7$ & 81,4 & 75,0 \\
\hline 30 & $34,4 \pm 3,1$ & $31,3 \pm 2,0$ & $13,07 \pm 0,9$ & $11,9 \pm 1,4$ & $15,2 \pm 1,1$ & $14,1 \pm 1,1$ & 83,1 & 84,8 \\
\hline 60 & $34,1 \pm 2,7$ & $32,3 \pm 1,1$ & $13,47 \pm 1,2$ & $13,5 \pm 1,1$ & $15,8 \pm 1,3$ & $14,5 \pm 0,8$ & 87,2 & 87,5 \\
\hline 90 & $29,1 \pm 2,0$ & $31,7 \pm 2,1$ & $12,79 \pm 0,8$ & $13,3 \pm 2,0$ & $13,1 \pm 1,3$ & $14,0 \pm 1,5$ & 87,2 & 86,6 \\
\hline 120 & $31,1 \pm 2,3$ & $27,4 \pm 2,7$ & $15,01 \pm 1,3$ & $14,2 \pm 1,7$ & $13,8 \pm 1,2$ & $11,5 \pm 1,8$ & 90,1 & 89,0 \\
\hline 150 & $25,2 \pm 1,5$ & $25,6 \pm 2,4$ & $12,90 \pm 1,3$ & $14,0 \pm 1,8$ & $9,8 \pm 1,4$ & $10,3 \pm 1,2$ & 97,1 & 87,2 \\
\hline
\end{tabular}

$\mathrm{R}=$ Recipientes. $\mathrm{P}=$ Película. Los resultados representan los valores medios de 6 repeticiones. ${ }^{1}$ Índice de Oscurecimento. 
en la luminosidad y en la tonalidad amarilla y roja de nueces americanas almacenadas durante 157 días, que resultaron en el oscurecimiento superficial de las mismas.

\subsection{Análisis sensorial}

Los resultados del análisis sensorial están sumarizados en la Tabla 6 . A través del análisis de regresión, se constata una reducción lineal altamente significativa $(p<0,0001)$ en las notas atribuídas a todas las características sensoriales durante el período de almacenamiento. El análisis de contrastes muestra que las notas de aceptabilidad no presentan diferencias significativas entre los dos tipos de envase ( $p \leq 0,05$ y $R^{2} \geq 0,7969$ ).

Valores de $R^{2}$ superiores a 0,60 en análises sensoriales e instrumentales en función del tiempo son considerados excelentes (De Marchi et al. , 2003). Estos resultados indican que la aplicación de vacío no contribuyó para prolongar la vida de anaquel de las nueces.

Considerando que 6,0 fue considerada como la nota límite para una calidad aceptable en las nueces, se puede concluir que estas muestran una calidad adecuada por 4 meses (120 días), ya que todos los atributos obtuvieron notas inferiores a 6,0 a los 150 días.

El analísis sensorial mostró una alta correlación lineal negativa con el índice de oscurecimiento (IO), siendo $R^{2}=-0,99$ para las nueces almacenadas en recipientes de polipropileno y $R^{2}=-0,6$ para las películas de nilón-polietileno al vacío.

\subsection{Calidad microbiológica de las nueces}

Durante el período de almacenamiento de las nueces de 150 días, no fue detectada la presencia de Salmonella sp. y los recuentos de coliformes a $45^{\circ} \mathrm{C}$ fueron menores que $3 \mathrm{NMP} / \mathrm{g}$. Estos resultados cumplen con los límites establecidos por la legislación brasileña, que determina la ausencia de Salmonella y un máximo de $10^{2} \mathrm{NMP} / \mathrm{g}$ para coliformes a $45^{\circ} \mathrm{C}$ (Brasil, 2001). En la Tabla 7, son mostrados los resultados del recuento total de hongos
Tabla 7

Recuento total de hongos y levaduras en nueces almacenadas en recipientes plásticos de polipropileno y en película de nilón-polietileno al vacío

\begin{tabular}{ccc}
\hline \multirow{2}{*}{ Tiempo (días) } & \multicolumn{2}{l}{ Hongos y levaduras (UFC/g) } \\
\cline { 2 - 3 } & Recipientes & Película \\
\hline 0 & $1,0 \times 10^{2}$ & $1,2 \times 10^{2}$ \\
30 & $1,0 \times 10^{2}$ & $3,0 \times 10^{2}$ \\
60 & $1,0 \times 10^{2}$ & $1,0 \times 10^{2}$ \\
90 & $1,0 \times 10^{2}$ & $1,0 \times 10^{2}$ \\
120 & $1,5 \times 10^{2}$ & $1,0 \times 10^{2}$ \\
150 & $1,5 \times 10^{2}$ & $1,0 \times 10^{2}$ \\
\hline
\end{tabular}

y levaduras. Estos valores son considerados bajos e indican que los envases utilizados fueron eficientes para garantizar la calidad microbiológica de las nueces.

Con base en los resultados obtenidos, se puede concluir que las nueces Pecán son una excelente fuente de energía, además de proveer altos contenidos de proteínas, minerales y fibras. La vida de anaquel de este producto fue determinada como siendo 120 días y fue independiente del envase utilizado en este estudio.

\section{AGRADECIMIENTOS}

Los autores agradecen a CAPES (Coordenação de Aperfeiçoamento de Pessoal de Nível Superior), a Divinut Indústria de Nozes Ltda. y a los componentes del panel sensorial por su valiosa contribución.

\section{BIBLIOGRAFÍA}

Alves MAO, Arruda CSA, Ogliari PJ, Meinert EM, Teixeira E, Barrera-Arellano D, Block JM. 2005. Efecto de la adición del absorbedor UV (Tinuvin 234) sobre la calidad del aceite de soja en envases de polietileno tereftalato (PET). Grasas y Aceites 53 (4) 245-253.

Tabla 6

Medias de aceptación de nueces almacenadas en recipientes plásticos de polipropileno y en película de nilón-polietileno al vacío

\begin{tabular}{|c|c|c|c|c|c|c|c|c|c|c|c|c|}
\hline \multirow{2}{*}{$\begin{array}{c}\text { Tiempo } \\
\text { (días) }\end{array}$} & \multicolumn{2}{|c|}{$\mathrm{AG}^{2}$} & \multicolumn{2}{|c|}{ Color } & \multicolumn{2}{|c|}{ Apariencia } & \multicolumn{2}{|c|}{ Olor } & \multicolumn{2}{|c|}{ Sabor } & \multicolumn{2}{|c|}{ Textura } \\
\hline & $\mathbf{R}$ & $\mathbf{P}$ & $\mathbf{R}$ & $\mathbf{P}$ & $\mathbf{R}$ & $\mathbf{P}$ & $\mathbf{R}$ & $\mathbf{P}$ & $\mathbf{R}$ & $\mathbf{P}$ & $\mathbf{R}$ & $P$ \\
\hline 0 & 8,00 & 8,15 & 8,03 & 8,09 & 7,91 & 8,09 & 7,83 & 8,07 & 7,74 & 8,35 & 7,83 & 7,97 \\
\hline 30 & 7,69 & 7,51 & 8,06 & 7,63 & 7,87 & 7,72 & 7,90 & 7,87 & 7,91 & 7,78 & 8,03 & 7,90 \\
\hline 60 & 7,13 & 7,87 & 7,20 & 7,83 & 7,43 & 7,77 & 7,53 & 7,61 & 7,03 & 7,74 & 7,53 & 7,88 \\
\hline 90 & 7,16 & 6,87 & 6,71 & 6,47 & 7,10 & 6,67 & 6,88 & 6,93 & 6,81 & 6,63 & 7,03 & 6,37 \\
\hline 120 & 5,93 & 6,27 & 6,39 & 6,54 & 6,03 & 6,04 & 6,03 & 6,27 & 5,64 & 6,50 & 6,39 & 6,61 \\
\hline 150 & 5,00 & 5,74 & 4,86 & 5,61 & 5,03 & 5,74 & 5,41 & 5,96 & 4,59 & 5,30 & 4,31 & 5,13 \\
\hline
\end{tabular}

$\mathrm{R}=$ Recipientes. $\mathrm{P}=$ Película. $\mathrm{n}=30 . \mathrm{AG}=$ aceptabilidad global . 
AOAC 2005. Association of Official Analytical Chemists. Official methods of analysis of the AOAC. 18th Ed. AOAC, Arlington, VA.

AOCS. 2003. Official Methods and Recommended Practices of the American Oil Chemist's Society, 15th Ed., AOCS Press, Champaign (II).

APHA 2001. American Public Health Association. Compendium of Methods for the Microbiological Examination of Foods, 4th Ed., APHA. Washington, D.C.

Brasil. Ministério da Agricultura, Pecuária e Abastecimento. 2007. www.agricultura.gov.br, 12/01/2007.

Brasil - Agência Nacional de Vigilância Sanitária - Ministério da Saúde. 2001. Resolução RDC n 12, de 02 de Janeiro de 2001. Regulamento técnico sobre padrões microbiológicos para alimentos. Diário Oficial da União, Brasília.

Buera MP, Lozano RD, Petriella, C. 1985. Definition of colour in the nonenzymatic browning process. Die Farbe $32 / 33$, 316-326.

Chun J, Lee J, Eitenmiller RR. 2005. Vitamine E and stability during storage of raw and dry roasted peanuts packaged under air and vacuum. Journal of Food Science 70 (4) 292-297.

Chun J, Lee J, Eitenmiller R. 2002. Effects of variety and crop year on tocopherols in pecan. Journal of Food Science 67, 1356-1359.

Crews C, Hough P, Godward P, Brereton P, Lees M, Guiet S, Winkelmann, W. 2005. Study of the main constituents of some authentic walnut oils. Journal of Agricultural and Food Chemistry 53, 4853-4860.

De Marchi R, Monteiro M, Cardello HMB. 2003. Avaliação da vida-de-prateleira de um isotônico natural de maracujá (Passiflora edulis Sims. f. flavicarpa Deg.). Brazilian Journal of Food Technology 6 (2) 292-300.

Erickson MC, Santerre CR, Malingre ME. 1994. Oxidative stability in raw and roasted pecans: chemical, physical and sensory measurements. Journal of Food Science 59 (6) 1234-1239.

Firestone, D. 1999. Physical and Chemical Characteristics of Oils, Fats and Waxes. AOCS Press, Washington, DC.

Forbus Jr WR, Sente, SD, Lyon BG, Dupuy HP. 1980. Correlation of objective and subjective measurements of pecan kernel quality. Journal of Food Science 45, 1376-1379.

Gamli ÖF, Hayoglu I. 2007. The effect of the different packaging and storage conditions on the quality of pistachio nut paste. Journal of Food Engineering 78 (2) 443-448.

García-Pascual P, Mateos M, Carbonell V, Salazar DM. 2003. Influence of storage conditions on the quality of shelled and roasted almonds. Biosystens Engineering 84 (2) 201-209.
Kolakowska A. 2003. Lipid Oxidation in Food Systems en Sikorski ZE, Kolakowska A. (Eds) Chemical and Functional Properties of Food Lipids. 133-165. CRC Press, London, UK.

Kornsteiner M, Wagner KH, Elmadfa I. 2006. Tocopherols and total phenolics in 10 different nut types. Food Chenistry 98, 381-387.

Maskan M, Karatas S. 1999. Storage stability of wholesplit pistachio nuts (Pistachia vera L.) at various conditions. Food Chemistry 66, 227-233.

Meilgaard M, Civille GV, Carr BT. 1999. Affective Tests: Consumer Test and In-House Panel Acceptance Tests en Sensory Evaluation Techniques, 3rd Ed., 231-263. CRC Press, London.

Munõz AM, Civille VG, Carr BT. 1992. Sensory evaluation in quality control, Van Mostrand, Reinhold USA.

Ortiz ERN. 2000. Propriedades nutritivas e nutracêuticas das nozes. (Monografia) - Especialização em Tecnologia de Alimentos, Universidade de Santa Cruz do Sul, RG, Brasil. 49 p.

Shahidi F. Miraliakbari H. 2005. Tree Nut Oils en Shahidi F. (Ed). Bailey's Industrial Oil and Fat Products 3, 6th Ed. John Wiley \& Sons, Inc. Hoboken, NJ.

Singanusong R, Mason RL, D'arcy BRD. 2003. Compositional changes of Australia-grown western schley pecans [Carya illinoinensis (Wangenh.) K. Koch] during maturation. Journal of Agricultural and Food Chemistry 51, 406-412.

United States Department of Agriculture - USDA. National Nutrient Database for Standard Reference, Release 19 (2006), www.nal.usda.gov/fnic/foodcomp/ search, 21/04/2007.

Valenzuela AB, Nieto SK. 2001. Los antioxidantes: protectores de la calidad en la industria alimentaria. $\mathrm{Li}$ bro $10^{\circ}$ Aniversario. Recopilación de Artículos Técnicos de 1990-2000. ASAGA- Asociación Argentina de Grasas y Aceites. 1-41, 85-94.

Venkatachalam, M. 2004. Chemical composition of select pecan [Carya illinoinensis (Wangenh.) K. Koch] varieties and antigenic stability of pecan proteins. (Dissertation) - College of Human Sciences, The Florida State University.

Villarreal-Lozoya JE, Lombardini L, Zevallos LC. 2007. Phytochemical constituents and antioxidant capacity of different pecan [Carya illinoinensis (Wangenh.) K. Koch] cultivars. Food Chemistry 102 (4) 1241-1249.

Wakeling LT, Mason RL, D'arcy BR, Caffin NA. 2001. Composition of pecan cultivars Wichita and Western Schley [Carya illinoinensis (Wangenh.) C. Koch] grown in Australia. Journal of Agricultural and Food Science 49, 1277-1281.

Recibido: 5/10/07 Aceptado: 27/11/07 